\title{
Lars O. Erikson, Metafact: Essayistic Science in Eighteenth-Century France
}

\section{Peter Balazs}

\section{(2) OpenEdition}

10 Journals

\section{Édition électronique}

URL : http://journals.openedition.org/studifrancesi/30261

DOI : 10.4000/studifrancesi.30261

ISSN : 2421-5856

Éditeur

Rosenberg \& Sellier

\section{Édition imprimée}

Date de publication : 1 avril 2006

Pagination : 152-153

ISSN : 0039-2944

\section{Référence électronique}

Peter Balazs, "Lars O. Erikson, Metafact: Essayistic Science in Eighteenth-Century France », Studi

Francesi [En ligne], 148 (XLX | I) | 2006, mis en ligne le 30 novembre 2015, consulté le 19 avril 2021.

URL : http://journals.openedition.org/studifrancesi/30261 ; DOI : https://doi.org/10.4000/

studifrancesi.30261

Ce document a été généré automatiquement le 19 avril 2021.

\section{(c)}

Studi Francesi è distribuita con Licenza Creative Commons Attribuzione - Non commerciale - Non opere derivate 4.0 Internazionale. 


\title{
Lars O. Erikson, Metafact: Essayistic Science in Eighteenth-Century France
}

\author{
Peter Balazs
}

\section{RÉFÉRENCE}

LARS O. ERIKSON, Metafact: Essayistic Science in Eighteenth-Century France, Chapel Hill, North Carolina Studies in the Romance Languages and Litteratures, 2004, pp. 194.

1 Le livre d'inspiration foucaldienne d'E. Erickson analyse les conditions de l'établissement des faits scientifiques et des traits caractéristiques d'une certaine écriture essayistique dont il recherche les traces dans les ouvrages de Maupertuis, de Diderot et de Fontenelle. Erickson affirme que les années 1750-1800 constituent une véritable période de transition, porteuse d'une transformation épistémologique d'importance. D'accord sur ce point avec Foucault, l'auteur américain argue que l'épistémé classique est remplacée par l'épistémé moderne, mais, contrairement à l'auteur des Mots et les choses, il ne croit pas à la possibilité d'une transformation brusque et ultra-rapide. Le livre, consacré aux aspects multiples de cette transformation épistémologique majeure, postule que l'essai, par son indétermination fondamentale, est un genre particulièrement bien adapté aux méta-considérations (c'est-a-dire à la réflexion sur la possibilité de la connaissance) très à la mode tout au long de cette période pendant laquelle les faiblesses de l'épistémé classique (celle du mécanisme et de la représentation illimitée) commencent déjà à se manifester, alors que la nouvelle épistémé (moderne) n'est pas encore opératoire. La crise de l'ancienne se manifeste a plusieurs niveaux: après la dissolution apparente de l'objectivité cartésienne, Diderot et Maupertuis (les deux protagonistes du livre) se voient obligés à se consacrer à la question de la médiation et au problème de la langue.

2 Autour des années 1750, le doute envahit la science: l'universalisme des idées innées dont la validité est garantie par la bonne volonté du Créateur ne tient plus. Afin d'obtenir une connaissance stable, l'on doit avoir recours à des sources extérieures, tel 
le sauvage hypothétique de Rousseau, la statue de Condillac ou le persan de Montesquieu: l'homme doit reculer d'un pas et regarder soi-même en étranger, puisqu'il est incapable de percevoir les vrais moteurs de la nature directement, uniquement par les sens. La perte de l'immédiateté et le changement de paradigme scientifique expliquent l'importance croissante de la réflexion méthodologique.

3 L'essai, genre littéraire spécifique (Bacon) et manière d'écrire (Montaigne) est particulièrement adapté à l'expression des idées inédites. Quoique les textes analysés par Erickson ne portent pas le mot «essai» dans leurs titres, notre auteur y identifie la présence d'une écriture essayistique. Les avantages de cette écriture sont nombreux: accessible aux penseurs qui se trouvent à l'écart des cercles savants et académiques, elle ne vise pas un public déterminé, elle permet l'expression de l'incertitude de l'auteur qui ne présente pas à ses lecteurs des vérités indiscutables, mais plutôt le processus d'établissement de la vérité. L'auteur de l'essai est conscient de la subjectivité de son expérience personnelle, mais se demande comment celle-ci pourrait aspirer à une validité universelle. Cet aspect de l'écriture essayistique n'est pas sans rapport avec l'objectif de vulgarisation: la science étant avant tout une question de méthode, l'utilisation d'une méthodologie adaptée au défi donne à chacun la possibilité de faire des expériences. La connaissance scientifique ne peut donc pas demeurer l'affaire réservée à une élite de savants, les dames semi-cultivées peuvent désormais s'y intéresser. Dans les périodes de transition, les philosophes et les savants cherchent volontiers l'appui des milieux extra-scientifiques, afin d'obtenir de meilleures positions dans le monde scientifique à venir.

4 L'interrogation «metafactuelle», c'est-a-dire la réflexion sur les conditions de la production des faits scientifiques n'appartient ni à l'épistémé classique, ni à l'épistémé moderne: les penseurs analysés par Erickson se rendent compte que la connaissance scientifique ne peut s'ancrer que dans les sensations empiriques de l'homme. Ils savent également que l'homme sensible fait en même temps partie de la nature qu'il entend observer. La vraie connaissance est donc celle qui est d'origine subjective, mais objectivable par l'intermédiaire de procédés intellectuels de grande complexité.

5 Le dernier chapitre du livre analyse le rôle et la position de la littérature dans cette seconde moitié du XVIII ${ }^{e}$ siècle et ultérieurement. L'épistémé classique, forte de l'idée de la représentation illimitée, crut à l'unité fondamentale des lettres, tandis que les modernes ont séparé la science (qui recherche le vrai et l'utile) de la littérature (à la poursuite $\mathrm{du}$ beau). La science essayistique occupe une position particulièrement fragile entre ces deux conceptions, et Erickson se sert de cette affirmation pour montrer que l'opposition de la science et de la vérité n'est que le produit contingent d'un moment historique particulier. 